\title{
Nanocartography in the Age of Automated TEM
}

Matthew Olszta ${ }^{1}$ and Kevin Fiedler ${ }^{2}$

${ }^{1}$ Pacific Northwest National Laboratory, Richland, Washington, United States, ${ }^{2}$ Washington State University, Richland, Washington, United States

Electron microscopy is no different from any other high-end, advanced scientific field that will someday be controlled by complex, automated algorithms. The question then becomes, how do we as a field accept and embrace automation? Since its inception, transmission electron microscopy has seen a number of instrumentational advancements that have taken an already complex technique and further propelled it into a realm where few can fully achieve full mastery. The knowledge gap created by this complexity has been often been filled with peripheral analysis programs that are most often black box in nature. The data received is only good as the knowledge being put in. So too will this be true of automated TEM if there is not a paradigm shift before the advancement of computer algorithms so advanced that automation becomes the next in a long line of black box techniques.

To combat this inevitable future of fully push-button microscopy, we have developed a methodology which can not only smoothly transition into the coming age of computer assisted analysis but also act as a teaching tool for early stage microscopists: NanoCartography. The advent of digital data collection was the first step in microscopy forgetting its place as a science and becoming a rudimentary (but highly technical) tool. Instead of logging each region of interest or tilt condition on paper to correlate to a marker on cellulose film, rapid digital data collection and reliance on perceived "meta" data has provided a latent sense of security for the next generation of microscopists. Our research seeks to overcome these challenges by providing a pathway for each analysis to be considered its own experiment, and combines not only dictation tools but as well crystallographic and tilt predictors to solve the most complex of materials issues at the nanoscale.

Whereas there have been others who have posited similar derivations over the past 3 decades, they have almost been entirely focused on simple cubic systems or consider a priori knowledge of a given crystal system $^{1-2}$. NanoCartography provides a means to not only explore known systems, but more importantly unknown systems thereby allowing for more analytical flexibility. In whole, the methodology of NanoCartography seeks to bring science back into the realm of all electron microscopy, not just high-end development of new techniques. Users of any experience level can optimize their experiments and strengthen collaborations with the larger microscopy community.

Automation is the wave of the future and many companies, especially microfabrication, are already instituting it for rapid, repeatable failure analyses. In order to better facilitate the eventual transition of all of electron microscopy, it is necessary to inject science back into an unclear future of fully automated, black box analysis. Every sample should be considered its own unique experiment that can be repeated by anyone, anytime, anywhere. NanoCartography will provide that smooth transition, and in the short term provide more optimal and accurate microscopic analysis.

\section{References}

1. Desktop Microscopist is a trademark of Desktop Microscopist; Jim Stanley.

2. Cautaerts, N., R. Delville, and D. Schryvers, ALPHABETA: a dedicated open-source tool for calculating TEM stage tilt angles. Journal of Microscopy, 2018. 273 